PDES, SUBMANIFOLDS AND

AFFINE DIFFERENTIAL GEOMETRY

BANACH CENTER PUBLICATIONS, VOLUME 57

INSTITUTE OF MATHEMATICS

POLISH ACADEMY OF SCIENCES

WARSZAWA 2002

\title{
CENTROAFFINE DIFFERENTIAL GEOMETRY AND ITS RELATIONS TO HORIZONTAL SUBMANIFOLDS
}

\author{
LUC VRANCKEN \\ Laboratoire de Mathématiques, LAMATH, ISTV2, Université de Valenciennes \\ 59313 Valenciennes Cedex 9, France \\ E-mail: luc.vrancken@web.de
}

\begin{abstract}
We relate centroaffine immersions $f: M^{n} \rightarrow \mathbb{R}^{n+1}$ to horizontal immersions $g$ of $M^{n}$ into $S_{n+1}^{2 n+1}(1)$ or $H_{n}^{2 n+1}(-1)$. We also show that $f$ is an equiaffine sphere, i.e. the centroaffine normal is a constant multiple of the Blaschke normal, if and only if $g$ is minimal.
\end{abstract}

1. Introduction. In the present paper, we study centroaffine spheres $M^{n}$ in $\mathbb{R}^{n+1}$ and horizontal immersions $M^{n}$ in $S_{n+1}^{2 n+1}(1)$ and $H_{n}^{2 n+1}(-1)$. The basic existence and uniqueness theorems for those submanifolds together with the necessary preliminaries are derived in Sections 2 and 3. As it turns out, both are essentially determined by a symmetric tensor field on $M^{n}$ satisfying certain properties. In Section 4, under the additional assumption that $M^{n}$ is simply connected, we use then these existence and uniqueness theorems to relate the two types of submanifolds. This allows us to translate theorems obtained about centroaffine spheres to corresponding theorems about horizontal immersions.

In particular for surfaces $M^{2}$ we obtain a relation between negative definite centroaffine spheres with vanishing Tchebychev form (elliptic affine spheres in the terminology of [4]) and minimal 'horizontal 'isometric immersions of $M^{2}$ in $S_{3}^{5}(1)$. Notice that, from the basic formulas derived in Section 3, such immersions always have ellipse of curvature a circle. More surprisingly however is that also non-horizontal minimal immersions can play a role in affine differential geometry. Indeed, it was shown in [3] how starting from an arbitrary minimal Riemannian immersion with ellipse of curvature a circle in $S_{3}^{5}(1)$, it is possible to construct a 3 -dimensional elliptic affine hypersphere.

2. Centroaffine differential geometry. First, we recall the basic facts about centroaffine hypersurfaces. However, in order to obtain in the definite case always a positive

2000 Mathematics Subject Classification: Primary 53A15.

Key words and phrases: affine differential geometry, affine hyperspheres, product structures.

Supported by a research fellowship of the Alexander von Humboldt Stiftung (Germany).

The paper is in final form and no version of it will be published elsewhere. 
definite metric, we slightly deviate from the standard approach. For more details and proofs see [6]. Let $M$ be an $n$-dimensional $C^{\infty}$-manifold and let $f: M \rightarrow \mathbb{R}^{n+1}$ be a non-degenerate hypersurface immersion whose position vector is nowhere tangent. Then $f$ can be regarded as a transversal field along itself and we call $\xi=-\epsilon f$ the centroaffine normal, where $\epsilon= \pm 1$. Following Nomizu, we call $f$ together with this normalization a centroaffine hypersurface. We get the centroaffine structure equations:

$$
\begin{aligned}
& D_{X} f_{*}(Y)=f_{\star}\left(\nabla_{X} Y\right)+h(X, Y) \xi, \\
& D_{X} \xi=-\epsilon f_{*}(X) .
\end{aligned}
$$

Here we choose $\epsilon=1$ unless the signature of the second fundamental form with respect to $f$ is less than $\frac{n}{2}$ in which case we choose $\epsilon=-1$. Here $D$ denotes the canonical flat connection of $\mathbb{R}^{n+1}, \nabla$ is a torsionfree connection on $M$, called the induced centroaffine connection, and $h$ is a non-degenerate symmetric (0,2)-tensor field, called the centroaffine metric. So $(M, h)$ is a semi-Riemannian manifold and the signature of the metric sign $h$ satisfies $\operatorname{sign} h \leq \frac{n}{2}$. The corresponding integrability conditions (equations of Gauss (3) and Codazzi (4)) are

$$
\begin{aligned}
& R(X, Y) Z=h(Y, Z) X-h(X, Z) Y, \\
& (\nabla h)(X, Y, Z)=(\nabla h)(Y, X, Z),
\end{aligned}
$$

where $(\nabla h)(X, Y, Z)=X h(Y, Z)-h\left(\nabla_{X} Y, Z\right)-h\left(Y, \nabla_{X} Z\right)$. If we define

$$
C(X, Y, Z)=(\nabla h)(X, Y, Z),
$$

we get a totally symmetric (0,3)-tensor field, called the cubic form.

Denote by $\hat{\nabla}$ the Levi-Civita connection of $h$, by $\hat{R}, \widehat{\operatorname{Ric}}$ and $\hat{\kappa}$ the curvature tensor, the Ricci tensor and the normalized scalar curvature of $\hat{\nabla}$, respectively. The difference tensor $K$, which is defined by

$$
K_{X} Y=K(X, Y)=\nabla_{X} Y-\hat{\nabla}_{X} Y,
$$

is a symmetric tensor field related to the cubic form by $C(X, Y, Z)=-2 h\left(K_{X} Y, Z\right)$. Hence for every $X, K_{X}$ is selfadjoint with respect to $h$. Furthermore, we define the Tchebychev form $T$, the Tchebychev vector field $T^{\sharp}$ and the Pick invariant $J$ by

$$
\begin{aligned}
& n T(X)=\operatorname{trace} K_{X}, \\
& h\left(T^{\sharp}, X\right)=T(X), \\
& 4 n(n-1) J=4 h(K, K)=h(C, C) .
\end{aligned}
$$

We call $M$ an equiaffine sphere if and only if the Tchebychev form vanishes identically. It is well known that in that case, the centroaffine normal is a constant multiple of the Blaschke equiaffine normal. It is also well known that

$$
\begin{aligned}
& h\left(K_{X} Y, Z\right)=h\left(Y, K_{X} Z\right), \\
& \hat{R}(X, Y) Z=K_{Y} K_{X} Z-K_{X} K_{Y} Z+\epsilon(h(Y, Z) X-h(X, Z) Y), \\
& (\hat{\nabla} K)(X, Y, Z)=(\hat{\nabla} K)(Y, X, Z), \\
& \hat{\kappa}=J+\epsilon-\frac{n}{n-1} h\left(T^{\sharp}, T^{\sharp}\right),
\end{aligned}
$$

where $\epsilon$ is as defined before. Then, we have the following existence theorem: 
THEOREM 1. Let $(M, h)$ be an $n$-dimensional simply connected semi-Riemannian manifold with $\operatorname{sign} h \leq \frac{n}{2}$. Let $\epsilon= \pm 1$ and let $K$ be a symmetric tensor field on $M$ satisfying (9), (10), (11). Then there exist a centroaffine immersion $f: M^{n} \rightarrow \mathbb{R}^{n+1}$ with $\xi=-\epsilon f$ as affine normal, $h$ as affine metric and $\nabla=K+\widehat{\nabla}$, where $\widehat{\nabla}$ is the Levi Civita connection of the metric $h$, as induced affine connection. Moreover, the immersion $f$ is unique up to centroaffine transformations.

Proof. In order to obtain the existence part, we define a connection $\nabla$ on $M$ by

$$
\nabla_{X} Y=K(X, Y)+\widehat{\nabla}_{X} Y
$$

where $\widehat{\nabla}$ is the Levi Civita connection of the semi-Riemannian metric $h$. Then, we have that

$$
\begin{aligned}
(\nabla h)(X, Y, Z)= & X h(Y, Z)-h\left(\nabla_{X} Y, Z\right)-h\left(Y, \nabla_{X} Z\right) \\
= & h\left(\widehat{\nabla}_{X} Y, Z\right)+h\left(Y, \widehat{\nabla}_{X} Z\right)-h(K(X, Y), Z) \\
& \quad-h\left(\widehat{\nabla}_{X} Y, Z\right)-h(Y, K(X, Z))-h\left(Y, \widehat{\nabla}_{X} Z\right) \\
= & -2 h(K(X, Y), Z) .
\end{aligned}
$$

Hence by $(9)$ we deduce that $(\nabla h)(X, Y, Z)$ is totally symmetric. Next, we have that

$$
\begin{aligned}
R(X, Y) Z= & \nabla_{X} \nabla_{Y} Z-\nabla_{Y} \nabla_{X} Z-\nabla_{[X, Y]} Z \\
= & K\left(X, \nabla_{Y} Z\right)+\widehat{\nabla}_{X} \nabla_{Y} Z-K\left(Y, \nabla_{X} Z\right) \\
& \quad-\widehat{\nabla}_{Y} \nabla_{X} Z-K([X, Y], Z)-\widehat{\nabla}_{[X, Y]} Z \\
= & K(X, K(Y, Z))-K(Y, K(X, Z))+\hat{R}(X, Y) Z \\
& \quad+(\widehat{\nabla} K)(X, Y, Z)-(\widehat{\nabla} K)(Y, X, Z) \\
= & \epsilon(h(Y, Z) X-h(X, Z) Y) .
\end{aligned}
$$

Finally, we define the affine shape operator by $S=\epsilon I$. Applying now the general existence theorem of [2], we obtain that there exists an affine immersion $f: M^{n} \rightarrow \mathbb{R}^{n+1}$ and a transversal vector field $\xi$ such that we can write

$$
\begin{aligned}
& D_{X} f_{*} Y=f_{*} \nabla_{X} Y+h(X, Y) \xi, \\
& D_{X} \xi=-f_{*} S X=-\epsilon X .
\end{aligned}
$$

Since $D_{X}(f+\epsilon \xi)=0$, we obtain that if necessary by applying a translation, we may assume that $f$ is centroaffine.

Next, we show the uniqueness part. Let $f_{1}, f_{2}: M^{n} \rightarrow \mathbb{R}^{n+1}$ be two immersions satisfying the assumptions of the theorem. Applying the uniqueness theorem [1], see also [2], we have that $f_{1}$ and $f_{2}$ are related by an affine transformation. It then follows from (1) and the definition of $\xi$ that the translation component is zero. Hence $f_{1}$ and $f_{2}$ are related by a centroaffine transformation.

3. Horizontal submanifolds. We denote by $p=\left(x_{1}, y_{1}, x_{2}, y_{2}, \ldots, x_{n+1}, y_{n+1}\right)$ a point of $\mathbb{R}^{2 n+2}$ and define a product structure $P$ on $\mathbb{R}^{2 n+2}$ by

$$
P\left(x_{1}, y_{1}, \ldots, x_{n+1}, y_{n+1}\right)=\left(y_{1}, x_{1}, \ldots, y_{n+1}, x_{n+1}\right) .
$$


We then introduce a metric $\langle.,$.$\rangle on \mathbb{R}^{2 n+2}$ by

$$
\left\langle\left(v_{1}, w_{1}, \ldots, v_{n+1}, w_{n+1}\right),\left(\tilde{v}_{1}, \tilde{w}_{1}, \ldots, \tilde{v}_{n+1}, \tilde{w}_{n+1}\right)\right\rangle=\sum_{i=1}^{n+1} v_{i} \tilde{v}_{i}-\sum_{i=1}^{n+1} w_{i} \tilde{w}_{i} .
$$

Hence $\operatorname{sign}\langle.,\rangle=.n+1$. It immediately follows from (13) that for vectors $x$ and $y$ we have that

$$
\langle P x, P y\rangle=-\langle x, y\rangle
$$

In particular this implies that

$$
\langle x, P x\rangle=-\left\langle P x, P^{2} x\right\rangle=-\langle P x, x\rangle .
$$

Hence

$$
\langle x, P x\rangle=0 .
$$

We also deduce that $\left(D_{V} P\right) W=0$, where $D$ is the standard connection on $\mathbb{R}_{n+1}^{2 n+2}$. Let $S_{n+1}^{2 n+1}(1)$ and $H_{n}^{2 n+1}(-1)$ be the spaces respectively defined by

$$
S_{n+1}^{2 n+1}(1)=\left\{p \in \mathbb{R}^{2 n+2} \mid\langle p, p\rangle=1\right\}
$$

and

$$
H_{n}^{2 n+1}(-1)=\left\{p \in \mathbb{R}^{2 n+2} \mid\langle p, p\rangle=-1\right\} .
$$

It immediately follows that $S_{n+1}^{2 n+1}(1)$ is an umbilical hypersurface of $\mathbb{R}^{2 n+2}$, with $-p$ as normal and that the signature of the induced metric is $n+1$. Similarly, it follows that also $H_{n}^{2 n+1}(-1)$ is an umbilical hypersurface with normal $p$ and with signature of the induced metric equal to $n$. We denote by $\tilde{M}(1)=S_{n+1}^{2 n+1}(1)$ and by $\tilde{M}(-1)=H_{n}^{2 n+1}(-1)$. We now say that an immersion $f: M^{n} \rightarrow \tilde{M}(\epsilon)$, where $\epsilon= \pm 1$, is horizontal if and only if $P f$ is orthogonal to $f_{*} T_{p} M$. Notice that it follows from (15) that $P f$ is always a tangent vector field to $\tilde{M}(\epsilon)$. Decomposing the connection $D$ into a tangent component, a normal component which is tangent to $\tilde{M}(\epsilon)$ and a component in the direction of the position vector, we see that

$$
\begin{gathered}
D_{X} f_{*} Y=f_{\star}\left(\nabla_{X} Y\right)+\alpha(X, Y)-\langle X, Y\rangle \epsilon f, \\
D_{X} \eta=-f_{*} A_{\eta} X+\nabla_{X}^{\perp} \eta,
\end{gathered}
$$

where $X, Y$ are tangent vector fields and $\eta$ is a normal vector field to $M$ tangent to $\tilde{M}(\epsilon)$. The symmetric bilinear form $\alpha$ is the second fundamental form of the immersion $f$ in $\tilde{M}(\epsilon)$ and $\nabla^{\perp}$ is the normal connection of the immersion $f$ in $\tilde{M}(\epsilon)$. Since for a horizontal immersion, we have that $\langle P f, X\rangle=0$, for any tangent vector field $X$, we deduce that

$$
0=\left\langle P f_{*}(Y), X\right\rangle+\langle P f, \alpha(X, Y)\rangle .
$$

From (14) it follows that the first term is skewsymmetric in $X$ and $Y$, whereas the second term is symmetric. Hence we obtain that

$$
\left\langle P f_{*}(X), Y\right\rangle=0, \quad\langle P f, \alpha(X, Y)\rangle=0 .
$$

Hence $A_{P f}=0$ and $P$ maps the tangent space into the normal space of $M$ in $\tilde{M}(\epsilon)$. Therefore the normal space to $M$ in $\tilde{M}(\epsilon)$ is spanned by $P f$ and $P f_{*} T M$. Deriving the first equation of (3), and identifying $M$ with its image in $\mathbb{R}_{n+1}^{2 n+2}$ we deduce that

$$
0=\langle P X, \alpha(Y, Z)\rangle-\left\langle A_{P X} Z, Y\right\rangle \text {. }
$$


Since $P$ is parallel with respect to $D$, we deduce that

$$
0=\left(D_{X} P\right) f=D_{X} P f-P D_{X} f=\nabla_{X}^{\perp} P f-P X .
$$

Hence

$$
\nabla_{X}^{\perp} P f=P X
$$

Similarly, we get that

$$
\begin{aligned}
0 & =\left(D_{X} P\right) Y=D_{X} P Y-P D_{X} Y, \\
& =\nabla_{X}^{\perp} P Y-A_{P Y} X-P \nabla_{X} Y-P \alpha(X, Y)+\epsilon\langle X, Y\rangle P f .
\end{aligned}
$$

Hence,

$$
\nabla_{X}^{\perp} P Y=P \nabla_{X} Y-\epsilon\langle X, Y\rangle P f, \quad A_{P Y} X=-P \alpha(X, Y) .
$$

Finally, we recall that the equations of Gauss, Codazzi and Ricci for submanifolds of a semi-Riemannian space form state that

$$
\begin{aligned}
& R(X, Y) Z=\epsilon(\langle Y, Z\rangle X-\langle X, Z\rangle Y)+A_{\alpha(Y, Z)} X-A_{\alpha(X, Z)} Y, \\
& (\nabla \alpha)(X, Y, Z)=(\nabla \alpha)(Y, X, Z), \\
& \left\langle R^{\perp}(X, Y) \eta_{1}, \eta_{2}\right\rangle=\left\langle\left[A_{\eta_{1}}, A_{\eta_{2}}\right] X, Y\right\rangle .
\end{aligned}
$$

We introduce a symmetric bilinear form $\beta$ on $M$ by

$$
\beta(X, Y)=-P \alpha(X, Y)=A_{P Y} X=A_{P X} Y .
$$

We also write $\beta_{X} Y=\beta_{Y} X=\beta(X, Y)$. From (18) it follows that

$$
\langle\beta(X, Y), Z\rangle \text { is symmetric in } X, Y \text { and } Z \text {. }
$$

It now follows from (19) and (3) that we can rewrite

$$
\begin{aligned}
R(X, Y) Z & =\epsilon(\langle Y, Z\rangle X-\langle X, Z\rangle Y)-A_{P A_{P Y} Z} X+A_{P A_{P X} Z} Y \\
& =\epsilon\left(\langle Y, Z\rangle X-\langle X, Z\rangle Y-A_{P X} A_{P Y} Z+A_{P Y} A_{P X} Z\right. \\
& =\epsilon(\langle Y, Z\rangle X-\langle X, Z\rangle Y)-\beta(X, \beta(Y, Z))+\beta(Y, \beta(X, Z)) \\
& =\epsilon(\langle Y, Z\rangle X-\langle X, Z\rangle Y)-\left[\beta_{X}, \beta_{Y}\right] Z
\end{aligned}
$$

From (19), (3) and the Codazzi equation it follows that

$$
\begin{aligned}
(\nabla \beta)(X, Y, Z) & =\nabla_{X} \beta(Y, Z)-\beta\left(\nabla_{X} Y, Z\right)-\beta\left(X, \nabla_{Y} Z\right) \\
& =-\nabla_{X} P \alpha(Y, Z)+P \alpha\left(\nabla_{X} Y, Z\right)+P \alpha\left(Y, \nabla_{X} Z\right) \\
& =-P \nabla_{X}^{\perp} \alpha(Y, Z)+\epsilon\langle X, P \alpha(Y, Z)\rangle f+P \alpha\left(\nabla_{X} Y Z\right)+P \alpha\left(Y, \nabla_{X} Z\right) \\
& =-P(\nabla \alpha)(X, Y, Z)-\epsilon\langle P X, \alpha(Y, Z)\rangle .
\end{aligned}
$$

Since $\langle P X, \alpha(Y, Z)\rangle=\langle X, \beta(Y, Z)\rangle$ is totally symmetric in $X, Y$ and $Z$, we deduce that $\nabla \beta$ is totally symmetric if and only if $\nabla \alpha$ is totally symmetric. We now can formulate the basic existence and uniqueness theorems for horizontal submanifolds.

THEOREM 2. Let $f_{1}, f_{2}: M \rightarrow \tilde{M}(\epsilon)$ be two isometric horizontal immersions. Suppose that $\beta^{f_{1}}=\beta^{f_{2}}$. Then there exists an isometry $A$ of $\tilde{M}(\epsilon)$ such that $A f_{1}=f_{2}$. 
Proof. Let $p \in M$. We define an isometry $A_{p}$ of $\tilde{M}(\epsilon)$ by

$$
\begin{array}{ll}
A_{p} f_{1}(p)=f_{2}(p), & A_{p} f_{1 *}(X(p))=f_{2 *}(X(p)), \\
A_{p} P f_{1}(p)=P f_{2}(p), & A_{p} P f_{1 *}(X(p))=P f_{2 *}(X(p)) .
\end{array}
$$

Now, we show that $A$ is independent of the choice of $p$. Using the previous equations, it follows that

$$
\begin{aligned}
\left(D_{X} A\right) f_{1} & =D_{X}\left(A f_{1}\right)-A D_{X} f_{1}=D_{X} f_{2}-A f_{1 *} X=0, \\
\left(D_{X} A\right)\left(P f_{1}\right) & =D_{X}\left(P f_{2}\right)-A D_{X}\left(P f_{1}\right)=P f_{2 *} X-A P f_{1 *} X=0, \\
\left(D_{X} A\right)\left(f_{1 *}(Y)\right) & =D_{X} f_{2 *} Y-A D_{X} f_{1 *} Y \\
& =f_{2 *}\left(\nabla_{X} Y\right)+\alpha^{f_{2}}(X, Y)-A f_{1 *}\left(\nabla_{X} Y\right)-A \alpha^{f_{1}}(X, Y) \\
& =-P f_{2 *}\left(\beta^{f_{2}}(X, Y)\right)+A P f_{1 *}\left(\beta^{f_{1}}(X, Y)\right)=0, \\
\left(D_{X} A\right)\left(P f_{1 *} Y\right) & =D_{X} P f_{2 *}(Y)-A D_{X} P f_{1 *} Y=P D_{X} f_{2 *}(Y)-A P D_{X} f_{1 *} Y \\
& =P \alpha^{f_{2}}(X, Y)-A P \alpha^{f_{1}}(X, Y)=-f_{2 *} \beta^{f_{2}}(X, Y)+A f_{1 *} \beta^{f_{1}}(X, Y)=0 .
\end{aligned}
$$

This completes the proof of the theorem.

TheOrem 3. Let $(M,\langle.,\rangle$.$) be a simply connected semi-Riemannian manifold. Let \beta$ be a symmetric bilinear vector valued 2 form on $M$ such that

(i) $\langle\beta(X, Y), Z\rangle$ is totally symmetric,

(ii) $\nabla \beta$ is totally symmetric,

(iii) $R(X, Y) Z=\epsilon(\langle Y, Z\rangle X-\langle X, Z\rangle Y)-\left[\beta_{X}, \beta_{Y}\right] Z$.

Then there exists a horizontal isometric immersion $f: M \rightarrow \tilde{M}(\epsilon)$ with second fundamental form $\alpha$ such that $\beta(X, Y)=-P \alpha(X, Y)$.

Proof. We define a bundle $N M$ over $M$ by $N M=T M \oplus \mathbb{R} \oplus \mathbb{R}$ and we define a mapping $P: T M \oplus N M \rightarrow T M \oplus N M$ by

$$
\begin{aligned}
P(X, 0,0,0) & =(0, X, 0,0) \\
P(0, X, 0,0) & =(X, 0,0,0) \\
P(0,0,1,0) & =(0,0,0,1) \\
P(0,0,0,1) & =(0,0,1,0),
\end{aligned}
$$

a section $f=0 \oplus 0 \oplus 1$ of $N M$. We introduce a metric on $N M$ by

$$
\begin{array}{lll}
\langle P X, P Y\rangle=-\langle X, Y\rangle & \langle P X, f\rangle=0 & \langle X, P f\rangle=0, \\
\langle X, f\rangle=0 & \langle P X, P f\rangle=0 & \langle X, P Y\rangle=0, \\
\langle P f, P f\rangle=-\epsilon & \langle P f, f\rangle=0 & \langle f, f\rangle=\epsilon .
\end{array}
$$

Next, we define a connection $\nabla^{\perp}$ on $N M$ by

$$
\begin{aligned}
& \nabla_{X}^{\perp} P Y=P \nabla_{X} Y-\epsilon\langle X, Y\rangle P f, \\
& \nabla_{X}^{\perp} f=0, \quad \nabla_{X}^{\perp} P f=P X .
\end{aligned}
$$

Then we define a second fundamental form

$$
\gamma: T M \times T M \longrightarrow N M:(X, Y) \mapsto \gamma(X, Y)=-P \beta(X, Y)+\langle X, Y\rangle \epsilon f
$$


with corresponding Weingarten operators given by

$$
A_{P X} Y=\beta(X, Y), \quad A_{P f} Y=0, \quad A_{f} Y=-Y,
$$

for $X, Y$ tangent to $M$.

A straightforward computation then shows that the Gauss, Codazzi and Ricci equations are satisfied and hence by the existence and uniqueness theorem for immersions into real space forms, there exist an isometric immersion $g: M^{n} \longrightarrow \mathbb{R}_{n+1}^{2 n+2}$ with $\langle.,$.$\rangle as$ induced metric and a bundle isomorphism such that we can identify $N M$ with the normal bundle of $g$ in $\mathbb{R}_{n+1}^{2 n+2}$. Since $D_{X}(g-f)=0$, we see that after applying a translation, we may assume that $g=f$. Hence

$$
\langle g, g\rangle=\langle f, f\rangle=\epsilon,
$$

and $g\left(M^{n}\right) \subset \tilde{M}(\epsilon)$. Since $g=f$ is normal to $\tilde{M}(\epsilon)$, we deduce that the second fundamental form $\alpha$ of $M^{n}$ in $\tilde{M}(\epsilon)$ is given by $\alpha(X, Y)=-P \beta(X, Y)$.

Finally, in order to show that $M^{n}$ is horizontal, it is sufficient to show that the mapping $P$, defined in the beginning of the proof, is parallel along $M^{n}$. We have

$$
\begin{aligned}
\left(D_{X} P\right) Y & =D_{X} P Y-P D_{X} Y=\nabla_{X}^{\perp} P Y-A_{P Y} X-P \nabla_{X} Y-P \gamma(X, Y) \\
& =-\epsilon\langle X, Y\rangle P f-\beta(X, Y)+\beta(X, Y)+\epsilon\langle X, Y\rangle P f, \\
\left(D_{X} P\right) P Y & =D_{X} Y-P D_{X} P Y=P\left(P D_{X} Y-D_{X} P Y\right)=-P\left(D_{X} P\right) Y=0, \\
\left(D_{X} P\right) f & =D_{X} P f-P D_{X} f=P X-P X=0, \\
\left(D_{X} P\right) P f & =-P\left(D_{X} P\right) f=0 .
\end{aligned}
$$

4. A correspondence theorem. In this section, we relate the two types of submanifolds studied in the previous sections. First, we show:

THEOREM 4. Let $M$ be a simply connected manifold. Let $f: M^{n} \rightarrow \mathbb{R}^{n+1}$ be a centroaffine sphere with difference tensor $K$ and induced metric $h$. Then there exists a unique isometric horizontal immersion $g:\left(M^{n}, h\right) \rightarrow \tilde{M}(\epsilon)$ with second fundamental form $\alpha(X, Y)=-P K(X, Y)$.

Proof. The uniqueness part follows immediately from Theorem 2. To obtain the existence, we notice that (9), (10) and (11) state that $K$ is a symmetric bilinear form on the semi-Riemannian manifold $(M, h)$ satisfying the conditions of Theorem 3.

The converse is stated in the next theorem:

THEOREM 5. Let $M$ be a simply connected manifold. Let $g:\left(M^{n},\langle.\rangle.\right) \rightarrow \tilde{M}(\epsilon)$ be a horizontal isometric immersion with second fundamental form $\alpha$. Assume that index $\langle.,$. $\leq \frac{n}{2}$. Then there exist a unique $f: M \rightarrow \mathbb{R}^{n+1}$ centroaffine sphere such that the difference tensor $K$ satisfies $K=-P \alpha$.

Proof. The uniqueness part is clear from Theorem 1, whereas the existence part follows by combining (20), (24) and (28) with Theorem 1.

From the above theorems and (6) it is clear that $f$ is an equiaffine sphere if and only if $g$ is minimal. Applying the above theorems now for example to the results of [7], [8] and [4], we obtain the following corollaries: 
Corollary 1. Let $(M,\langle.,\rangle$.$) be a Riemannian manifold and let g: M^{n} \rightarrow S_{n+1}^{2 n+1}(1)$ be an isometric horizontal minimal immersion. If $M^{n}$ has constant sectional curvature, then $M^{n}$ is totally geodesic.

Corollary 2. Let $(M,\langle.,\rangle$.$) be a complete Riemannian manifold and g: M^{n} \rightarrow$ $S_{n+1}^{2 n+1}(1)$ a minimal isometric horizontal immersion. Then $M^{n}$ is totally geodesic.

Corollary 3. Let $(M,\langle.,\rangle$.$) be a Riemannian manifold and g: M^{n} \rightarrow H_{n}^{2 n+1}(-1)$ a minimal isometric horizontal immersion. Assume that $M^{n}$ has constant sectional curvature. Then either

(i) $M^{n}$ is totally geodesic, or

(ii) $g\left(M^{n}\right)$ is congruent to an open part of

$\left(\sinh u_{1}, \cosh u_{1}, \ldots, \sinh u_{n}, \cosh u_{n}, \sinh -\left(u_{1}+\ldots+u_{n}\right), \cosh -\left(u_{1}+\ldots+u_{n}\right)\right)$.

Similarly, the classification of minimal horizontal semi-Riemannnian immersions with constant sectional curvature follows from the proof of the Magid-Ryan conjecture in [8].

\section{References}

[1] F. Dillen, Equivalence theorems in affine differential geometry, Geom. Dedicata 32 (1989), 81-92.

[2] F. Dillen, K. Nomizu and L. Vrancken, Conjugate connections and Radon's theorem in affine differential geometry, Monatsh. Math. 109 (1990), 221-235.

[3] M. Kriele, C. Scharlach and L. Vrancken, A class of elliptic equiaffine 3-spheres in $\mathbb{R}^{4}$, Hokkaido Math. J. 30 (2001), 1-23.

[4] A. M. Li, U. Simon and G. Zhao, Global Affine Differential Geometry of Hypersurfaces, de Gruyter Exp. Math. 11, Walter de Gruyter, Berlin, 1993.

[5] K. Nomizu and T. Sasaki, Affine Differential Geometry, Cambridge Univ. Press, Cambridge, 1991.

[6] U. Simon, A. Schwenk-Schellschmidt, H. Viesel, Introduction to the Affine Differential Geometry of Hypersurfaces, Lecture Notes, Science Univ. Tokyo, 1991.

[7] L. Vrancken, A. M. Li and U. Simon, Affine spheres with constant affine sectional curvature, Math. Z. 206 (1991), 651-658.

[8] L. Vrancken, The Magid-Ryan conjecture for equiaffine hyperspheres with constant sectional curvature, J. Differential Geometry 54 (2000), 99-138. 